\title{
Corresponding Security Level with the Risk Factors of Personally Identifiable Information through the Analytic Hierarchy Process
}

Iuon-Chang Lin ${ }^{1}, 2^{*}$, Yung-Wang $\mathrm{Lin}^{3}$, Yu-Syuan $\mathrm{Wu}^{1}$

1 Department of Computer Science and Engineering, National Chung Hsing University, Taichung, Taiwan.

2 Department of Photonics and Communication Engineering, Asia University, Taichung, Taiwan.

${ }^{3}$ Department of Computer Science and Information Engineering, Asia University, Taichung, Taiwan.

* Corresponding author. Tel.: +886-4-22840864; email: iclin@nchu.edu.tw

Manuscript submitted March 15, 2015; accepted July 3, 2015.

doi: 10.17706/jcp.11.2.124-131

\begin{abstract}
Since Taiwan government has announced the New Version of Personal Information Protection Act , People began to pay attention to their personal information and privacy. Many industries significantly increased their responsibilities and faced more serious challenges. In order to cope with the requirements of the new law, BS10012 specification can help enterprises to reduce impact of personal data protection law. Enterprises need to rethink policy objectives, and make risk assessment of personally identifiable information (PII). This study using analytic hierarchy process (AHP) to identify the security level of PII. Corresponding security level with the risk factors of personal information, so that find the suitable countermeasure to protect users PII. Rarely see research about risk assessment of PII by using AHP, therefore the feasibility of the application of AHP in this regard is worthwhile to explore.
\end{abstract}

Keywords: BS10012, personally information, new version of personal information protection act.

\section{Introduction}

With the development of information technology, and the prevalence of network. A lot of information is transmitted through the network, which contains personal information. However, security issues related to information technology constantly occur, such as internal staff negligence leads to personal information loss within the enterprise, and confidential information is stolen. In 1995, the Taiwanese government had already promulgated the "Computer-Processed Personal Data Protection Act" [1], [2], that is the old Personal information Protection Act. With the evolution of the times, many of the provisions already not applicable to the protection of personal information privacy and interest, and people are growing emphasis on their personal information and privacy, so that the new version of Personal Information Protection Act appears in Taiwan [3].

The new version of Personal Information Protection Act expanded the scope of data protection, so that many industries significantly increased their legal liabilities and faced more serious challenges. In order to respond to the new law, many companies adopted BS10012: Personal Information Management System (PIMS) standard to reduce the impact of the New Version of Personal Information Protection Act [4]. The PIMS uses the PDCA (Plan-Do-Check-Action) process methodology and risk management [5], establish a complete management system for personal information protection. Risk management is one of the necessary processes for enterprises effectively manage and access user's personally identifiable 
information (PII). The one of risk management processes is risk assessment, this study using the analytic hierarchy (AHP) process [6] for risk assessment, to find security level [7] with the risk factors of personal information, rather than calculate the probability of risk occurrence. Corresponding security level with the risk factors of personal information, so that help managers to find the suitable countermeasures to protect users PII.

The architecture of this paper, Section 2 described research background and relevant work on risk assessment, Section 3 described method by using analytic hierarchy process (AHP) to measure risk factors, and corresponding security level. Section 4 discusses experiments and results, the fifth is the paper summation.

\section{Background and Related Work}

\subsection{New version of Personal Information Protection Act}

Taiwan has announced the New Version of Personal Information Protection Act since 2010 [3], and has been formally implemented in 1 October, 2012. There are several differences between the two Act, Applicable industry of New Personal Information Protection Act is not only restricted to the eight major industrial(credit information organizations, hospitals, schools, telecommunication businesses, financial businesses, securities businesses, insurance businesses, mass media), but also all industries are applicable. New Personal Information Protection Act where personal information means all personal data (all forms), not just computer processed personal information.

\subsection{BS 10012: PIMS}

BS 10012 specification was published in Britain in 2009, full name is Planning for a personal information management system (PIMS, BS10012: 2009), mainly aimed at protection of personal information. The PIMS uses the PDCA (Plan-Do-Check-Action) processes [5] methodology and risk management, establish a complete management system for the protection of personal information. Therefore many enterprises improved their IT environment security and strengthen the protection of personal information through PIMS. For establishment of a comprehensive management system for the protection of personal information must pass through the process of risk management.

The processes of risk management include doing risk identification, risk assessment, risk treatment, and risk monitoring down to do sequentially. To derive risk value on risk assessment phase, and to find ways to reduce or remove the risk on risk treatment phase. The phase of risk treatment to reduce the risk value to the enterprise accept range. If the risk value is not the acceptable range for enterprises, necessary to redo the risk assessment. If the risk value is the acceptable range for enterprises, regular audits and follow-up risk on risk monitoring phase.

\subsection{Risk Management}

Risk occurs because the weakness subjected the threats utilization [8], causing the enterprises damage (impact), the threats and vulnerabilities that are risk factors. Risk management through the identification of assets, threats and vulnerabilities, so that managers fully understand the risks location, and attempt to reduce the risk to an acceptable range, risk management help enterprises reduce accidents caused financial losses, and prevent potential hazards. The processes of risk management [9] include doing risk identification, risk assessment, risk treatment, and risk monitoring as shown in Fig. 1.

The processes of risk management include doing risk identification, risk assessment, risk treatment, and risk monitoring down to do sequentially. To derive risk value on risk assessment phase, and to find ways to reduce or remove the risk on risk treatment phase. The phase of risk treatment to reduce the risk value to 
the enterprise accept range. If the risk value is not the acceptable range for enterprises, necessary to redo the risk assessment. If the risk value is the acceptable range for enterprises, regular audits and follow-up risk on risk monitoring phase.

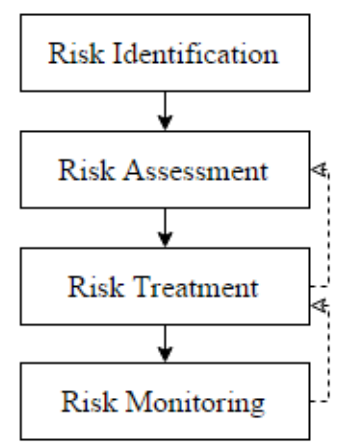

Fig. 1. The processes of risk management.

\subsection{Analytic Hierarchy Process (AHP)}

The AHP was proposed by Thomas L. Saaty in1960 [6]. When you want to make a decision with a problem, often need to consider multi-factor which will affect the final decision. The AHP takes into account the multi-factor of problems show that in the hierarchical architecture and pairwise comparisons. The AHP helps to simplify complex issues, and allows participants to subjective judgment and provide an objective decision [10]. So it is widely used, for example, be applied to risk assessment, decision alternatives, and program comparison, even can used in everyday life decisions. The hierarchical architecture is divided into three parts: top layer, intermediate layer, and lowest layer. Top layer represents the Goals of solve the problem, and focus of the problem analysis. Intermediate layer to identify the factors of the problem, the factors also called the Criteria, which impact the final decision-making. Lowest layer represents various measures or alternatives to solve the problem. And the following describes basic steps of AHP method [11]:

1) The decision participants define the problem to be evaluated.

2) Define the criteria which impact final decision of the problem, and each criteria can decompose into multiple subfactors, every criteria and subfactor are the elements.

3) To list the alternatives of the problem to be evaluated.

4) The above steps build in a top-down hierarchical architecture, corresponding to each level.

5) The elements of each layer pairwise comparison with 1-9 scale, and then obtain the comparison matrix, and calculate the eigenvalues and eigenvectors, over and over again to do until the last layer.

6) Doing consistency check to the matrix.

7) Assessing the weight of each layer and the overall weight, find best choice.

\section{Methodology}

This study uses the AHP in the risk assessment of personally identifiable information, and identify the security level of PII. The preliminary work what we need to do, first, we collected the literature relevant to risk assessment [7], [11]-[15], sorted out three categories of risk factors on Table 1, which caused risk of information assets disclosed or damaged, and the enterprises losses. Three categories of risk factors are human factors, environmental factors, and technological factors.

Second, doing risk assessment of these risk factors, in order to find the appropriate level of security by using AHP. The proposed risk assessment flowchart (Fig. 2) according to the following steps:

1) Define the goal of risk assessment in AHP, which is find security level in the study.

2) Define the criteria which is risk factors, impact final selection of security level. After identification the 
criteria, determining whether each principle subdivided into subfactors. Table 1 is what we classify risk factors and sub-factors.

Table 1. The Category of Risk Factors

\begin{tabular}{|c|l|l|l|}
\hline $\begin{array}{c}\text { Risk factors } \\
\text { (criteria) }\end{array}$ & Human factor & Environmental factor & Technological factor \\
\hline \multirow{3}{*}{ Subfactors } & $\begin{array}{l}\text { Data theft } \\
\text { Employees leaked data } \\
\text { Malicious viruses } \\
\text { Operation errors }\end{array}$ & $\begin{array}{l}\text { Politics/Law } \\
\text { Market demand } \\
\text { Power failure }\end{array}$ & $\begin{array}{l}\text { Equipment failure } \\
\text { Technology development }\end{array}$ \\
\hline
\end{tabular}

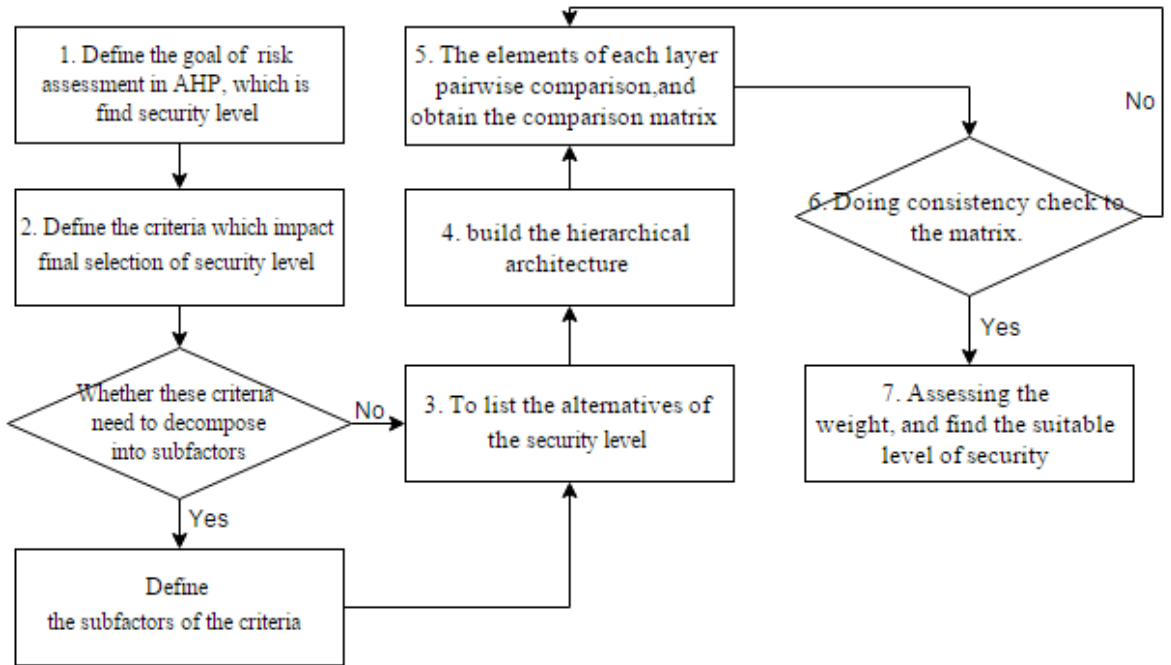

Fig. 2. The processes of risk management.

Table 2. The Elements Pairwise Comparison in Criteria Level

\begin{tabular}{|c|c|c|c|c|c|c|c|c|c|c|}
\hline Criterion & \multicolumn{9}{|c|}{ Scale } & Criterion \\
\hline \multirow{2}{*}{ E1 } & 9 & 7 & 5 & 3 & 1 & 3 & 5 & 7 & 9 & E2 \\
\hline & 9 & 7 & 5 & 3 & 1 & 3 & 5 & 7 & 9 & E3 \\
\hline E2 & 9 & 7 & 5 & 3 & 1 & 3 & 5 & 7 & 9 & E3 \\
\hline
\end{tabular}

3) Listing the options of the security level to correspond to the risk factors, we represent the security level in 1-3.

4) The hierarchical architecture was made out of the elements (risk factor, subfactors, and the security level) show in Fig. 3.

5) Pairwise comparison the elements of each layer with 1-9 scale, then obtain the comparison matrix, calculated the eigenvalues and eigenvectors, over and over again to do until the last layer. Obtained the relevant weights among elements.

a) Allow participants to assess and pairwise comparison the elements with 1-9 scale to define the importance among each element in same layer, and then obtained the comparison. Higher number means the greater degree of importance, represented by scale $1,3,5,7,9 . E$ indicates that the element parameters, $E_{i j}$ expressed as the element $E_{i}$ compare with the element $E_{j}$, if the scale is close to the more higher on the left, expressed the element $E_{i}$ is more importance than the element $E_{j}$. If the scale is 1 , means the element $E_{i}$ as important as the element $E_{j}$. Table 2 shows the elements pairwise comparison in criteria level, the decision makers can measure these risk factors, give rating.

b) After pairwise comparisons among the elements, obtained the comparison matrix. To calculate the eigenvalues and eigenvectors. If there were elements of $N$, would form a matrix $n^{*} n$ that is the comparison matrix, has the following characteristics: $E_{i j}>0, E_{i j}=\frac{1}{E_{j i}}$, and $E_{i i}=1$. So the comparison 
matrix also called the positive reciprocal matrix, like Table 3 is the comparison matrix of the element which we measure out in criteria level.

Table 3. RI Corresponds to the Order of the Matrix

\begin{tabular}{|l|l|l|l|l|l|l|l|l|l|}
\hline$N$ & 1 & 2 & 3 & 4 & 5 & 6 & 7 & 8 & 9 \\
\hline$R I$ & 0 & 0 & 0.58 & 0.90 & 1.12 & 1.24 & 1.32 & 1.41 & 1.45 \\
\hline
\end{tabular}

Table 4. The Comparison Matrix of the Criteria Level

\begin{tabular}{|l|l|l|l|l|}
\hline Criteria & Human & Environmental & Technological & S.G.M. $=4.477$ \\
$\lambda_{\max }=3.074$ \\
Human & 1.000 & 5.000 & 7.000 & 0.037 \\
Environmental & 0.200 & 1.000 & 3.000 & CR $=0.064$ \\
\hline Technological & 0.143 & 0.333 & 1.000 & \\
\hline
\end{tabular}

Doing consistency check to the comparison matrix. Each element divided by the sum of all elements in the some level, standardized for comparison matrix, and computed the eigenvectors, the consistency ratio (CR), consistency index (CI), and random index (RI). When $\mathrm{CR} \leqq 0.1$, means the matrix has consistency. If $\mathrm{CR}>0.1$, means the matrix has not consistency, redo the step5.

$$
\mathrm{CR}=\frac{\mathrm{CI}}{\mathrm{RI}}, \quad \mathrm{CI}=\frac{\lambda_{\max }-n}{n-1}
$$

$\lambda$ represents eigenvalues, $n$ represents the number of elements in the level. RI is the random consistency index, Saaty [6] noted RI is related to the matrix order $N$ as show in Table 3.

Evaluated the weight, obtained decision making, and found the suitable level of security. In accordance with different security levels, taken different security measures to effectively improve personal data protection in the organization. The security levels were divided into $1,2,3$, different security levels take different security measures. The higher number expressed the higher grade, need more attention, and more security mechanism.

\section{Experiments and Results}

Hypothesis we used the method in school case, to pairwise comparison the elements until the last layer on the hierarchical architecture show as Fig. 3, and give rating with 1-9 scale. Obtained the comparison matrix from the criteria level (shows in Table 4), and the subfactors level (shows in Tables 5, 6, 7). And then calculate the eigenvalues $(\lambda)$ of these matrices by calculating sum of geometric mean (S. G. M.) of column vector. After find the maximum eigenvalue of the comparison matrix, calculate $\mathrm{CI}$, and CR.

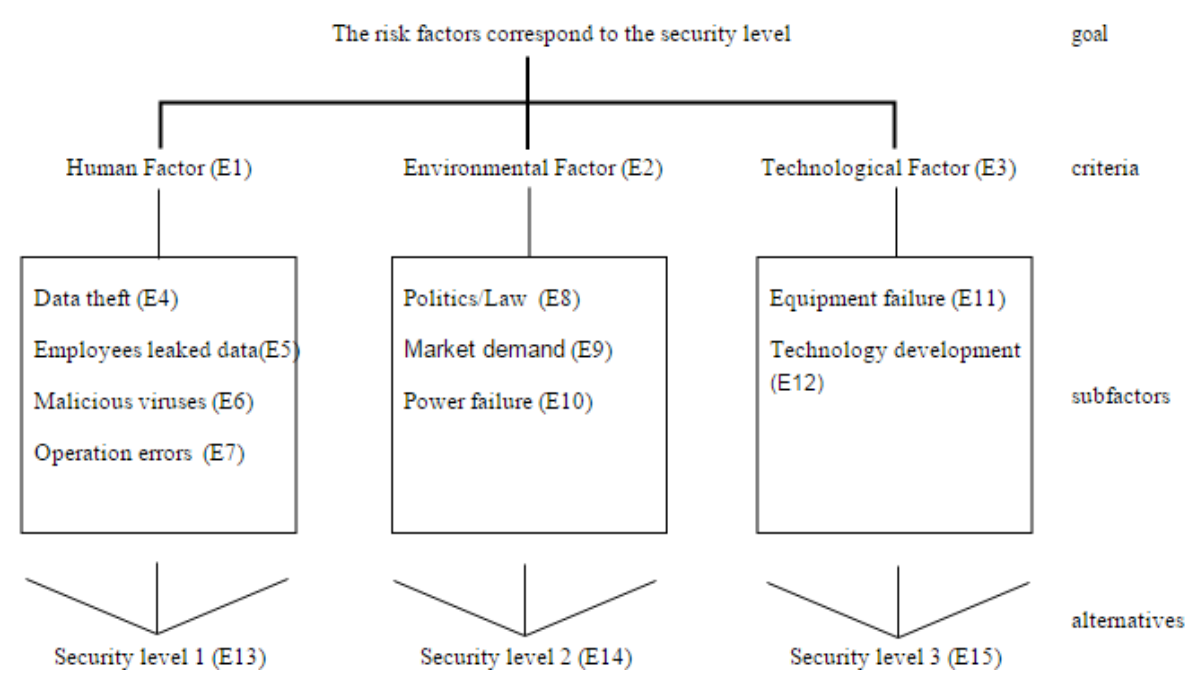

Fig. 3. The hierarchical architecture of risk factors. 
future, we hope the AHP method can be used widely on PII field of study.

\section{References}

[1] (1995). Taiwan Computer-Processed Personal Data Protection Law.

[2] Wang, E., H., H. (1999). ICT and economic development in Taiwan: Analysis of the evidence. Telecommunications Policy, 23(3), 235-243.

[3] (2010). Taiwan New version of Personal Information Protection Act.

[4] Liu, C., Y., Yang, S., S., \& Lin, I., C. (2014). Critical factors of educational institutions adoption for BS 10012: Persional information management system. International Journal of Network Security, 16(3), 161-167.

[5] Young, L. (2010). BS 10012: 2009 data protection-specification for a personal information management system. Records Management Journal, 20(1).

[6] Saaty, R. W. (1987). The analytic hierarchy process - what it is and how it is used. Mathematical Modelling, 9(3), 161-176.

[7] Chen, J., Wu, G., Shen, L., \& Ji, Z. (2011). Differentiated security levels for personal identifiable information in identity management system. Expert Systems with Applications, 38(11), 14156-14162.

[8] Farn, K. J., Lin, S. K., \& Fung, A. R. W. (2004). A study on information security management system evaluation - assets, threat and vulnerability. Computer Standards \& Interfaces, 26(6), 501-513.

[9] Humphreys, E. (2008). Information security management standards: Compliance, governance and risk management. Information Security Technical Report, 13(4), 247-255.

[10] Bantayan, N. C., \& Bishop, I. D. (1998). Linking objective and subjective modelling for landuse decision-making. Landscape and Urban Planning, 43(1), 35-48.

[11] Dey, P. K. (2010). Managing project risk using combined analytic hierarchy process and risk map. Applied Soft Computing, 10(4), 990-1000.

[12] Syamsuddin, I., \& Hwang, J. (2009, November). The application of AHP model to guide decision makers: A case study of e-banking security, Proceedings of IEEE Fourth International Conference on Computer Sciences and Convergence Information Technology (pp. 1469-1473).

[13] Pfleeger, C., P., \& Pfleeger, S., L. (2002). Security in computing. Prentice Hall Professional Technical Reference.

[14] Guan, B. C., Lo, C. C., Wang, P., \& Hwang, J., S. (2003, October). Evaluation of information security related risks of an organization: the application of the multicriteria decision-making method. Proceedings of IEEE 37th Annual 2003 International Carnahan Conference on Security Technology (pp. 168-175).

[15] Sendi, A., S., Jabbarifar, M., Shajari, M., \& Dagenais, M. (2010, May). FEMRA: Fuzzy expert model for risk assessment. Proceedings of IEEE Fifth International Conference on Internet Monitoring and Protection (ICIMP) (pp. 48-53).

[16] Zhao, D. M., Wang, J. H., \& Ma, J. F. (2006, August). Fuzzy risk assessment of the network security. Proceedings of IEEE International Conference on Machine Learning and Cybernetics (pp. 4400-4405).

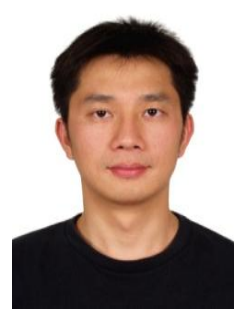

Iuon-Chang Lin received the Ph.D. in computer science and information engineering in March 2004 from National Chung Cheng University, Chiayi, Taiwan. He is currently a professor of the Department of Management Information Systems, National Chung Hsing University, Taichung, Taiwan. His current research interests include electronic commerce, information security, RFID information systems, and cloud computing. 
Yung-Wang Lin is a Ph.D. student of the Department of Computer Science and Information Engineering, Asia University, Wufeng Taichung Taiwan. His current research interests include information security and cloud computing. He also employed in NanKai University of Technology.

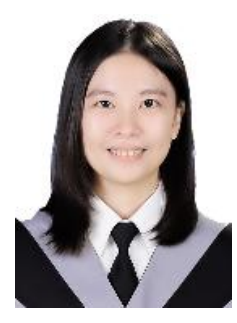

Yu-Syuan Wu was born on June 11, 1990. She graduated from National Chin-Yi University of Technology in Taiwan, and majored in the Department of Information Management. Now, she is a master degree student, studying in National Chung Hsing University in Taiwan. She has studied in the information security of personally identifiable information. 